OPEN ACCESS

Edited by:

Guobao Xu,

Chinese Academy of Sciences, China

Reviewed by:

Longhua Guo,

Jiaxing University, China

Matteo Mauro,

Université de Strasbourg, France

Shenguang $\mathrm{Ge}$,

University of Jinan, China

Guizheng Zou,

Shandong University, China

${ }^{*}$ Correspondence:

Jun-Jie Zhu

jizhu@nju.edu.cn

Specialty section: This article was submitted to Inorganic Chemistry, a section of the journal

Frontiers in Chemistry

Received: 17 November 2020 Accepted: 09 February 2021

Published: 19 March 2021

Citation:

Cao Y and Zhu J-J (2021) Recent Progress in Electrochemiluminescence of Halide Perovskites.

Front. Chem. 9:629830

doi: 10.3389/fchem.2021.629830

\section{Recent Progress in Electrochemiluminescence of Halide Perovskites}

\author{
Yue Cao and Jun-Jie Zhu* \\ State Key Laboratory of Analytical Chemistry for Life Science, School of Chemistry and Chemical Engineering, Nanjing University, \\ Nanjing, China
}

Halide perovskites are a rapidly developing class of solution-processable semiconductors which, to date, have a huge impact across several scientific communities. The remarkable photophysical attributes of halide perovskites illustrate their considerable potential in the electrochemiluminescence (ECL) realm. Over the past 4 years, great progress has been achieved in using halide perovskites as ECL emitters. In this mini-review, the basic characteristics, synthetic approaches, and ECL mechanisms for halide perovskite emitters are first introduced. To the best of our knowledge, most of the reported ECLactive halide perovskites and their disclosed unique features are detailly summarized. Stabilization and interface manipulation strategies for desirable ECL performance are further highlighted. The preliminary halide perovskites-related ECL applications are finally discussed, and prospects are also anticipated.

Keywords: electrochemiluminescence, stability, biosensing, interface manipulation, halide perovskite

\section{INTRODUCTION}

Electrochemiluminescence (ECL) is a light-emitting phenomenon arisen from electrochemical reactions between electrogenerated species in the vicinity of electrode. Known for excellence in low background, high sensitivity, and simple instrument, ECL has been acknowledged as a versatile analytical technology in life analysis, environmental monitoring, and pharmaceutical research, etc. (Ma et al., 2020). The emitters play a crucial role as electronic-to-optical transducers in ECL systems, whose exploitation and utilization are always the direction of efforts. Since the pioneering investigation on the ECL of silicon nanocrystals (NCs) (Ding et al., 2002), various ECL available semiconducting NCs have sprung up exuberantly, featuring a solution-processable colloidal state with quantum size effect, malleable surface chemistry, favorable optical property, and stable chemical composition (Miao, 2008).

The recent surge of interest in halide perovskites (PeNCs) has emerged in light-emitting diodes, lasers, and solar cells because of their appealing optoelectronic properties (Stoumpos and Kanatzidis, 2016). In 2016, Huang et al. first observed the ECL phenomenon during the study of all-inorganic $\mathrm{CsPbBr}{ }_{3} \mathrm{PeNCs}$, which opens the doors to the fundamental ECL research and application of PeNCs. After nearly 4 years of development, although various ECL-active PeNCs and a few unique merits have been disclosed, ECL research on PeNCs is still in its infancy and the subject of heightened concern. A recent review majors in the ECL performance of various PeNCs systems in either organic or aqueous media (Kong et al., 2020). In this mini-review, therefore, we present a detailed summary of the progress in using PeNCs as ECL emitters, which sketches the creative trajectory from the basic investigation of ECL-active PeNCs to the design strategies for desirable ECL property and resulting 
applications, hoping to provide readers with a comprehensive understanding of relevant contents and new ideas.

\section{BASIC CHARACTERISTICS AND SYNTHETIC APPROACHES}

PeNCs are characterized by the general formula $\mathrm{AMX}_{3}$, where $\mathrm{A}$ is a monovalent cation [e.g., $\mathrm{Cs}^{+}, \mathrm{CH}_{3} \mathrm{NH}_{3}{ }^{+}\left(\mathrm{MA}^{+}\right), \mathrm{HC}\left(\mathrm{NH}_{2}\right)_{2}{ }^{+}$ $\left.\left(\mathrm{FA}^{+}\right)\right], \mathrm{M}$ is a bivalent metal cation (e.g., $\mathrm{Pb}^{2+}, \mathrm{Ge}^{2+}, \mathrm{Sn}^{2+}$, etc.), and $\mathrm{X}$ is a halide anion $\left(\mathrm{Cl}^{-}, \mathrm{Br}^{-}, \mathrm{I}^{-}\right)$. Octahedral $\mathrm{MX}_{6}{ }^{4-}$ is formed by the coordination of $\mathrm{M}$ and $\mathrm{X}$ at its center and vertex, respectively. The crystal structure of PeNCs consists of anionic $3 \mathrm{D}$ networks of corner-sharing $\mathrm{MX}_{6}{ }^{2-}$ octahedra and the cavities occupied by A.

ECL emitters of PeNCs are mainly solution-processed colloidal NCs or quantum dots (QDs) via a hot-injection route (HIR) or a ligand-assisted reprecipitation method (LARM). Take the case of $\mathrm{CsPbX}_{3}$ NCs formation, HIR is conducted by swiftly injecting cesium oleate into an octadecene (ODE) solution containing $\mathrm{PbX}_{2}$, oleylamine $(\mathrm{OAm})$, and oleic acid $(\mathrm{OA})$ at high temperature $\left(>140^{\circ} \mathrm{C}\right.$ ) in a nitrogen atmosphere (Xue et al., 2017). In contrast, LARM is performed under mild reaction conditions without heating and nitrogen protection, which begins by dissolving precursors $\left(\mathrm{CsX}\right.$ and $\left.\mathrm{PbX}_{2}\right)$ in a "good" solvent (e.g., N, N-dimethylformamide, dimethylsulfoxide), and then transferring into a "poor" antisolvent (typically toluene) to reprecipitate NCs in the presence of ligands (Cao et al., 2020b). Besides, microwave was also utilized for one-step synthesis of $\mathrm{CsPbX}_{3} \mathrm{NCs}$ as ECL emitters via directly irradiating the ODE solution containing precursors $\left(\mathrm{Cs}_{2} \mathrm{CO}_{3}\right.$ and $\left.\mathrm{PbX}_{2}\right)$ and ligands (OA and $\left.\mathrm{OAm}\right)$ (Wang et al., 2020c).

The ECL mechanism of PeNCs is generally investigated following both annihilation and coreactant routes, which is basically consistent with the previously proposed nanomaterial ECL systems (Miao, 2008). The annihilation route transmits the ECL signal from single emitters via the direct transferring of exergonic electron between the electrogenerated reduced and oxidized radicals of PeNCs, which can be described as follows (Reactions 1-4):

$$
\begin{gathered}
\text { PeNCs }-\mathrm{e} \rightarrow \mathrm{PeNCs}^{+\bullet} \\
\text { PeNCs }+\mathrm{e} \rightarrow \mathrm{PeNCs}^{-} \\
\mathrm{PeNCs}^{+}{ }^{\bullet} \mathrm{PeNCs}^{-} \rightarrow \mathrm{PeNCs}^{*}+\mathrm{PeNCs}^{0} \\
\text { PeNCs }^{*} \rightarrow \mathrm{PeNCs}^{+} \mathrm{hv}
\end{gathered}
$$

The coreaction mechanism requires an appropriate coreactant to assist emitters for the ECL signal. Taking the example of tripropylamine (TPrA), a typical "oxidation-reduction" coreactant for PeQDs, the coreactant route can be described as the following Reactions 5-8. Briefly, TPrA undergoes electrooxidization and deprotonation to form a highly reductive radical intermediate $\operatorname{TPrA}{ }^{\bullet}$. Meanwhile, PeNCs are electro-oxidized to $\mathrm{PeNCs}^{+\bullet}$. The radiative recombination of $\mathrm{TPrA}^{\bullet}$ and $\mathrm{PeNCs}^{+\bullet}$ produces excited PeNCs* for ECL emission. Searching for promising coreactants is beneficial to expand potential ECL applications of PeNCs. As far as we know, PeNCs mainly emit anodic ECL with the coreactants including TPrA (Cai et al., 2018), 2-(dibutylamino)ethanol (DBAE) (Li et al., 2019), ascorbic acid (AA) (Cao et al., 2020c), ethyl acetate (EA) (Xue et al., 2017), and hydrogen peroxide $\left(\mathrm{H}_{2} \mathrm{O}_{2}\right)$ (Huang et al., 2017). Besides, the cathodic ECL of certain PeNCs was also observed in the presence of $\mathrm{K}_{2} \mathrm{~S}_{2} \mathrm{O}_{8}$ (Peng et al., 2020) or benzoyl peroxide (BPO) (Cao et al., 2019).

$$
\begin{gathered}
\text { TPrA }-\mathrm{e} \rightarrow \mathrm{TPrA}^{\bullet}+\mathrm{H}^{+} \\
\text {PeNCs }-\mathrm{e} \rightarrow \mathrm{PeNCs}^{+} \\
\mathrm{PeNCs}^{+\bullet}+\mathrm{TPrA}^{\bullet} \rightarrow \mathrm{PeNCs}^{*}+\text { Products } \\
\mathrm{PeNCs}^{*} \rightarrow \text { PeNCs }+ \text { hv }
\end{gathered}
$$

\section{AVAILABLE HALIDE PEROVSKITE EMITTERS}

Multifarious ECL-active PeNCs spring up vigorously, whose fundamental studies and practical uses have set off a new research upsurge. To the best of our knowledge, the main ECL parameters for the reported PeNCs-based systems are summarized in Table 1, and several representative ones are described in detail here.

Fully-inorganic $\mathrm{CsPbBr}_{3} \mathrm{NCs}$ are the most reported PeNCs as ECL emitters. Huang et al. (2016) first investigated the ECL behaviors of cubic $\mathrm{CsPbBr}_{3} \mathrm{NCs}(12-15 \mathrm{~nm})$. The direct charge injection produced various charged NCs, which further generated charge-transfer-mediated ECL in an annihilation or coreaction pathway (Figure 1A). The ECL spectrum of the $\mathrm{CsPbBr}_{3} \mathrm{NCs}$ displayed a sole and symmetric peak centered at $519 \mathrm{~nm}$, and the full width at half maximum (fwhm) was only about $20 \mathrm{~nm}$, which is much narrower than classical ECL luminophores, that is, for example, commercial $\mathrm{Ru}(\mathrm{bpy})_{3}{ }^{2+}$ over $100 \mathrm{~nm}$. Transient ECL signal occurred via the electrochemical oxidation of negativecharged NCs, and no ECL signal was obtained conversely, indicating the electrochemically switchable ECL property (Figure 1A $\mathbf{A}_{1}$ ).

Organometallic PeNCs with A as organic cations have attracted board interest in optoelectronic and photovoltaic devices. By using ECL technology, the redox and charge transfer natures of highly crystalline $\mathrm{MAPbBr}_{3}$ nanowires $(500$ $\times 50 \mathrm{~nm}$ ) were first studied in phosphate buffer solution (PBS) by Tan et al. (2017). MAPbBr 3 NCs could be electrochemically oxidized or reduced to several opposite charged states. The redox nature regulated the charge transfer process for ECL emission via an annihilation route or a coreaction route with TPrA or $\left(\mathrm{NH}_{4}\right)_{2} \mathrm{~S}_{2} \mathrm{O}_{8}$ as the coreactants (Figure 1B). Electrochemically switchable ECL was also observed, but transient ECL was only obtained via injecting electrons into the positive-charged NCs (Figure $\mathbf{1 B}_{1}$ ). Besides, their anodic ECL spectrum demonstrated a single and symmetric peak around $535 \mathrm{~nm}$ with high color purity (fwhm, $25 \mathrm{~nm}$ ), which was almost identical to the photoluminescence (PL) one. Soon after, anodic ECL of $\mathrm{FAPbBr}_{3} \mathrm{NCs}(13 \pm 2 \mathrm{~nm})$ centered at $534 \mathrm{~nm}$ with an fwhm of $31 \mathrm{~nm}$ was also discovered by injecting holes into the 
TABLE 1 | Main basic and ECL parameters for various PeNCs-based systems.

\begin{tabular}{|c|c|c|c|c|c|c|c|c|}
\hline PeNCs & Morphol. & Size nm & $\lambda_{\mathrm{PL}}{ }^{\mathrm{a}} \mathrm{nm}$ & Coreactant & $\lambda_{\mathrm{ECL}}{ }^{\mathrm{b}} \mathbf{n m}$ & fwhm $_{\mathrm{ECL}} \mathrm{nm}$ & $\phi_{\mathrm{ECL}}{ }^{\mathrm{c}} \%$ & Ref. \\
\hline $\mathrm{CsPbBr}_{3}$ & cube & $12-15$ & 515 & TPrA/BPO & 519 & 20 & - & Huang et al. (2016) \\
\hline $\mathrm{CsPbBr}_{3}$ & cube & $\sim 10-25$ & 516 & $\mathrm{H}_{2} \mathrm{O}_{2}$ & 518 & 20 & - & Huang et al. (2017) \\
\hline $\mathrm{CsPbBr}_{3}$ & cube & 8 & 509 & EA & 515 & 24 & 500 & Xue et al. (2017) \\
\hline $\mathrm{CsPbBr}_{3}$ & cube & $\sim 20$ & 518 & TPrA & $\sim 560$ & $\sim 41$ & - & Cai et al. (2018) \\
\hline $\mathrm{CsPbBr}_{3}$ & cube & $\sim 8$ & 500 & $\mathrm{~S}_{2} \mathrm{O}_{8}^{2-}$ & 570 & $\sim 62$ & 1.6 & Peng et al. (2020) \\
\hline $\mathrm{CsPbBr}_{3}$ & cube & 9 & 519 & EA & - & - & - & Hao et al. (2019) \\
\hline $\mathrm{CsPbBr}_{3}$ & cube & 9.57 & 509 & $\mathrm{AA}$ & 506 & $\sim 100$ & - & Wang et al. (2020b) \\
\hline $\mathrm{CsPbBr}_{3}$ & cube & 13 & 511 & TPrA & 569 & 26 & - & Qiu et al. (2019) \\
\hline $\mathrm{CsPbBr}_{3}$ & cube & $\sim 20$ & 520 & $\mathrm{H}_{2} \mathrm{O}_{2} / \mathrm{TPrA}$ & - & - & - & Wang et al. (2020c) \\
\hline $\mathrm{MAPbBr}_{3}$ & nanowire & $500 \times 50$ & 531 & $\mathrm{TPrA}_{2} \mathrm{O}_{8}{ }^{2-}$ & 535 & 25 & - & Tan et al. (2017) \\
\hline $\mathrm{FAPbBr}_{3}$ & cube & $13 \pm 2$ & 529 & TPrA & 534 & 31.3 & - & Wang et al. (2020a) \\
\hline $\mathrm{Cs}_{3} \mathrm{Bi}_{2} \mathrm{Br}_{9}$ & dot & $4.8 \pm 1.24$ & 389 & TPrA/BPO & - & - & - & Cao et al. (2019) \\
\hline $\mathrm{MAPbCl}_{1.08} \mathrm{Br}_{1.92}$ & dot & $3.5 \pm 0.15$ & 450 & TPrA & 473,744 & $\sim 41, \sim 200$ & - & Wusimanjiang et al. (2019) \\
\hline $\mathrm{Rb}_{0.2} \mathrm{Cs}_{0.8} \mathrm{PbBr}_{3}$ & cube & $10 \pm 2$ & 514 & DBAE & 510 & 20 & - & Chen et al. (2020) \\
\hline $\mathrm{Sb}^{3+}-\mathrm{CsPbBr}_{3}$ & cube & 21 & 520 & TPrA & 520 & 19 & - & Jia et al. (2019) \\
\hline $\mathrm{Ce}^{4+}-\mathrm{CsPbBr}_{3}$ & dot & 5 & 468 & TPrA & 522 & 26 & - & Fu et al. (2020b) \\
\hline $\mathrm{CsPbBr}_{3}-\mathrm{CeO}_{2}$ & column & $70 \times 12$ & 520 & IPTA & 522 & 20 & - & ru et al. (2UटU0) \\
\hline $\mathrm{CsPbBr}_{3}-\mathrm{TOP}$ & cube & $\sim 13$ & 523 & OAm, AA & 523 & $\sim 30$ & 57.08 & Cao et al. (2020b) \\
\hline $\mathrm{CsPbBr}_{3}-\mathrm{Ag}_{2} \mathrm{~S}$ & cube & $8-10$ & 515 & TPrA & $\sim 520$ & $\sim 50$ & & Fu et al. (2020a) \\
\hline $\mathrm{CsPbBr}_{3}-\mathrm{DBAE} @ \mathrm{SiO}_{2}$ & cube $^{\mathbf{d}}$ & $7.6^{\mathbf{d}}$ & 518 & DBAE & $\sim 530$ & $\sim 47$ & 410 & Li et al. (2019) \\
\hline $\mathrm{CsPbBr}_{3} @$ silica gel & cube $^{\mathbf{d}}$ & $19^{d}$ & 516 & DBAE & 517 & 39 & - & Li et al. (2020) \\
\hline $\mathrm{CsPbBr}_{3} @ \mathrm{HCNS}$ & - & - & 507 & $\mathrm{AA}$ & 525.7 & 34.5 & - & Cao et al. (2020c) \\
\hline $\mathrm{CsPbBr}_{3}-\mathrm{NCDs@HZIF-8}$ & cube $^{\mathbf{d}}$ & $\sim 4.0^{\text {d }}$ & 504 & NCDs & 539 & 41.3 & - & Cao et al. (2020a) \\
\hline
\end{tabular}

${ }^{a, b} \lambda_{P L}$ and $\lambda_{E C L}$ represent the maximum emission wavelength of $P L$ and ECL, respectively.

${ }^{c} \varphi_{E C L}$ represents ECL efficiency relative to the standard $R u(b p y){ }_{3}{ }^{2+} / T P r A$ system.

${ }^{d}$ the actual morphology and size of $\mathrm{CsPbBr}_{3}$ in hybrids.

negative-charged NCs or using the TPrA coreactant (Wang et al., 2020a).

Lead-based PeNCs are currently the best performing ECL emitters; however, the toxicity of lead hinders the commercial prospects. In theory, lead can be replaced with other low-toxic metals. Through an all-round improvement of LARM, Cao et al. (2019) synthesized lead-free $\mathrm{Cs}_{3} \mathrm{Bi}_{2} \mathrm{Br}_{9}$ QDs with good monodispersity, remarkable stability, and highly passivated surface, thereby affording unprecedented optical property with a PL quantum yield up to $37 \%$. The high-quality QDs were first discovered with both anodic and cathodic ECL emission in either an annihilation or a coreaction route (Figure 1C), which might open an avenue for the design of lead-free PeNCs as eco-friendly and stable ECL emitters.

Halogen anion adjustable is one of the outstanding characters of PeNCs, which can lead to bright PL emission over the entire visible spectral region. Wusimanjiang et al. (2019) synthesized mixed-halogen perovskite of $\mathrm{MAPbCl}_{1.08} \mathrm{Br}_{1.92}$ QDs $(3.5 \mathrm{~nm})$. Rare sky-blue ECL emission centered at $473 \mathrm{~nm}$ was first achieved, demonstrating the feasibility of halogen aniontunable ECL spectra. Besides, mixed-monovalent cations can also regulate the ECL properties of PeNCs. Chen et al. (2020) prepared $\mathrm{Rb}_{\mathrm{x}} \mathrm{Cs}_{1-\mathrm{x}} \mathrm{PbBr}_{3} \mathrm{NCs}$ by partly replacing $\mathrm{Cs}^{+}$with $\mathrm{Rb}^{+}$. With the increase of $\mathrm{Rb}^{+}$content, the ECL spectra blue shifted gradually, and the ECL intensity displayed an up-and-down trend. Heterovalent substitution is also proposed to tailor the optoelectrical attributes of PeNCs. Jia et al. (2019) directly introduced $\mathrm{Sb}^{3+}(\mathrm{Sb} / \mathrm{Pb}=1: 3$, molar ratio $)$ in the synthesizing procedure of $\mathrm{CsPbBr}_{3} \mathrm{NCs}$ via HIR. The partial replaces of $\mathrm{Pb}^{2+}-\mathrm{Br}$ bonds with strong $\mathrm{Sb}^{3+}-\mathrm{Br}$ bonds enlarged the band gap and preserved highly passivated surface states. Meanwhile, the doping behavior induced more vacancies and impurities, facilitating radiative charge transfer and electron injection/ transfer efficiency for both enhanced PL and ECL. Also, Fu et al. (2020b) reported a similar $\mathrm{Ce}^{4+}$ doping process and a $\mathrm{CeO}_{2}$-conversion strategy, achieving modulation of electrochemical and radiative-charge-transfer behaviors of $\mathrm{CsPbBr}_{3} \mathrm{NCs}$ for tunable PL and ECL. The facile and largescale tunability of ECL property via ions adjustable might open novel probabilities to design multicolor emitters.

\section{STABILIZATION STRATEGY FOR DESIRABLE ECL}

The inherent vulnerability of PeNCs toward the external environment severely restricted their ECL progress. Although significant advances have been achieved in enhancing the stability of PeNCs by surface engineering or embedding into inert matrices, these protective layers are at a compromise with charge and mass transports, which inevitably lower the ECL efficiency. Thus, many strategies have been proposed for suitable constructions in the trade-off between ECL efficiency and structural stability.

ECL is particularly sensitive to surface chemistry because charges must pass through the surface before being injected into NCs to trigger the ECL (Ding et al., 2002). Thus, surface modification with robust ligands can form a highly passivated surface of PeNCs. Cao et al. (2020b) synthesized surfacepassivated $\mathrm{CsPbBr}_{3}$ NCs by post-synthetic treatment with tri- 


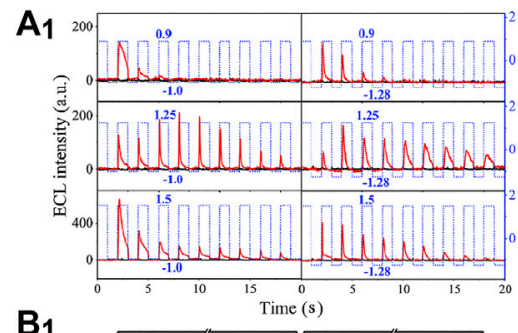

$B_{1}$

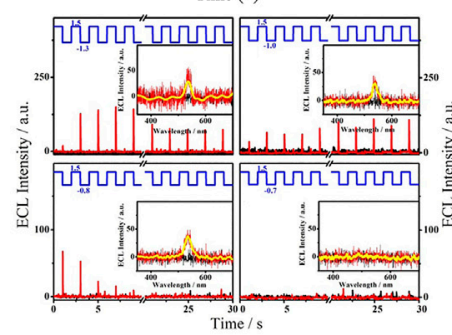

$\mathrm{C}_{1}$

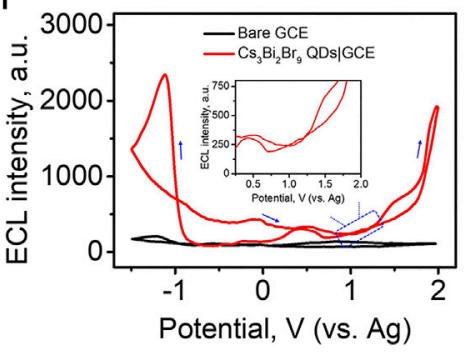

\section{A2}

$\mathrm{B}_{2}$
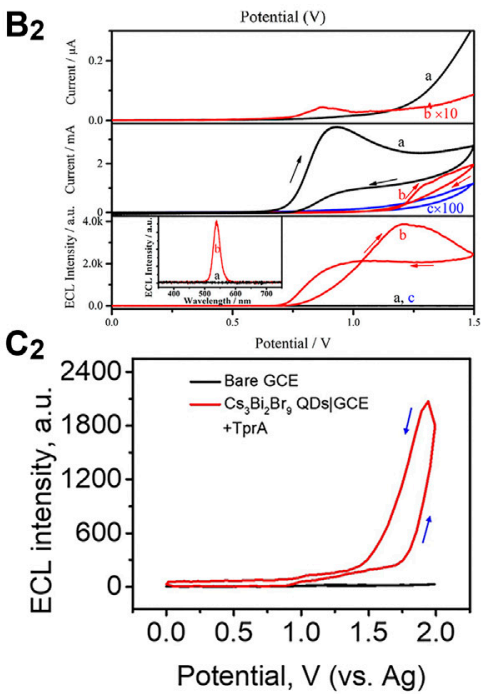

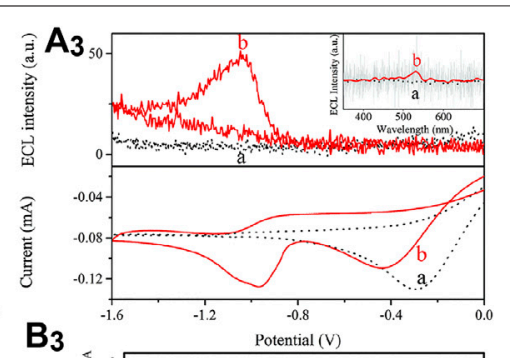

$\mathrm{B}_{3}$

\section{$\mathrm{C}_{3}$}

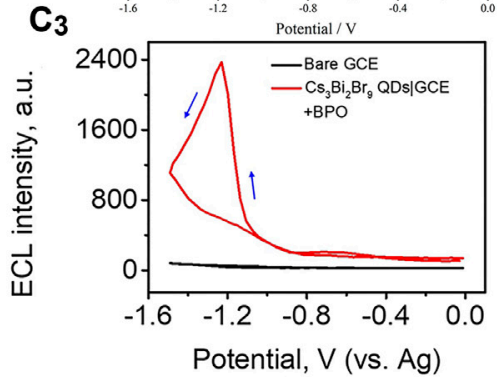

FIGURE 1 | ( $\mathbf{A}_{\mathbf{1}}$ ) Oxidation initiated transient electrochemiluminescence (ECL), $\left(\mathbf{A}_{\mathbf{2}}\right)$ anodic coreactant ECL (10 mA TPrA), and $\left(\mathbf{A}_{\mathbf{3}}\right)$ cathodic coreactant ECL (5 mM $\mathrm{BPO}$ ) of $\mathrm{CsPbBr}_{3} \mathrm{NCs} \mid \mathrm{GCE}$ in air-free dichloromethane containing $0.10 \mathrm{M}$ tetra- $n$-butylammonium hexafluorophosphate (TBAPF $F_{6}$ ). Reproduced with permission from

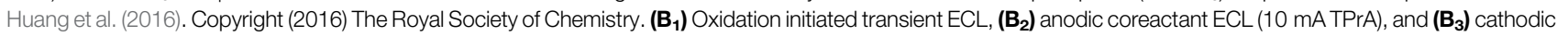
coreactant ECL [100 mM $\left(\mathrm{NH}_{4}\right)_{2} \mathrm{~S}_{2} \mathrm{O}_{8}$ ] of $\mathrm{MAPbBr}_{3} \mathrm{NCs} \mid \mathrm{GCE}$ in air-free $0.10 \mathrm{M}$ PBS. Reproduced with permission from Tan et al. (2017). Copyright (2017) American Chemical Society. $\left(\mathbf{C}_{1}\right)$ Annihilation ECL, $\left(\mathbf{C}_{2}\right)$ anodic coreactant ECL (10 mM TPrA), and $\left(\mathbf{C}_{3}\right)$ cathodic coreactant ECL (10 mM BPO) of Cs $\mathbf{B}_{3} \mathrm{Bi}_{2} \mathrm{Br}_{9} \mathrm{QDs} / \mathrm{GCE}$ in a binary organic solution of acetonitrile and toluene containing 0.05 M TBAPF 6 . Reproduced with permission from Cao et al. (2019). Copyright (2019) American Chemical Society.

n-octylphosphine (TOP) (Figure 2A). As a Lewis base and a highly branched ligand, TOP succeeded in reducing the NCs surface defects for enhanced ECL intensity and delaying the degradation of PeNCs. Passivating PeNCs surface with noble metal-based nanoparticles (NPs) can enhance the charge injection/transfer capacity while increasing stability. Fu et al. (2020a) introduced silver diethyldithiocarbamate into the $\mathrm{CsPbBr}_{3} \mathrm{NCs}$ crude solution. During the process, $\mathrm{Ag}_{2} \mathrm{~S}$ NPs were generated in situ on the $\mathrm{CsPbBr}_{3}$ NCs surface. The nanoheterostructure guaranteed enhanced aqueous stability and radiative charge transfer, thereby affording 9-fold ECL enhancement in an aqueous electrolyte.

Silica has been recognized as an inert and robust coating to enhance the stability of guests. Li et al. (2020) encapsulated $\mathrm{CsPbBr}_{3} \mathrm{NCs}$ in silica gel via injecting $\mathrm{CsBr}$ aqueous solution into $\mathrm{Cs}_{4} \mathrm{PbBr}_{6} \mathrm{NCs}$ and tetramethoxysilane (TMOS) hexane solution. The small amount of water triggered the conversion of $\mathrm{Cs}_{4} \mathrm{PbBr}_{6} \mathrm{NCs}$ to $\mathrm{CsPbBr}_{3} \mathrm{NCs}$ and the in-situ formation of waterproof silica gel. The abundant $\mathrm{CsBr}$ not only reduced the transformation rate but also greatly enhanced the conductivity of silica gel. Accordingly, the perovskite hybrids achieved strong ECL and still maintained their optical property after thirty-day storage in $\mathrm{CsBr}$ solution. To reduce the negative impact of silica coating on charge and mass transport, Li et al. (2019) coencapsulated $\mathrm{CsPbBr}_{3}$ QDs and coreactant into silica matrix through in-situ hydrolysis of TMOS (Figure 2B). DBAE was selected as the optimal coreactant because its tertiary amine could act as both coreactant for $\mathrm{CsPBrr}_{3}$ QDs and catalyst for TMOS hydrolysis, and its hydroxyl group could be cross-condensed with TMOS and interact with silanol groups via hydrogen bonding. The perovskite-derived ternary hybrids achieved multifold ECL efficiencies (vs. $\mathrm{Ru}(\mathrm{bPy})_{3}{ }^{2+} / \mathrm{TPrA}$ ) and preserved $55 \%$ of initial ECL intensity after $48 \mathrm{~h}$ storage in ambient condition with $100 \%$ relative humidity.

Semiconductor heterojunction can regulate the transfer and recombination of carriers in its hybrid structure. Cao et al. (2020c) prepared hollow $\mathrm{g}^{-} \mathrm{C}_{3} \mathrm{~N}_{4}$ nanospheres (HCNS) via polycondensation of cyanamide in a silica template, and $\mathrm{CsPbBr}_{3} \mathrm{NCs}$ further grew in situ within HCNS via HIR. In this work, HCNS as an ideal scaffold not only protected the internal $\mathrm{CsPbr}_{3} \mathrm{NCs}$ but also provided a matching band edge for upgraded ECL performance (Figure 2C).

Metal-organic frameworks (MOFs) are recognized as fulfilling nanocarriers, attributable to tunable porosity, high surface area, 


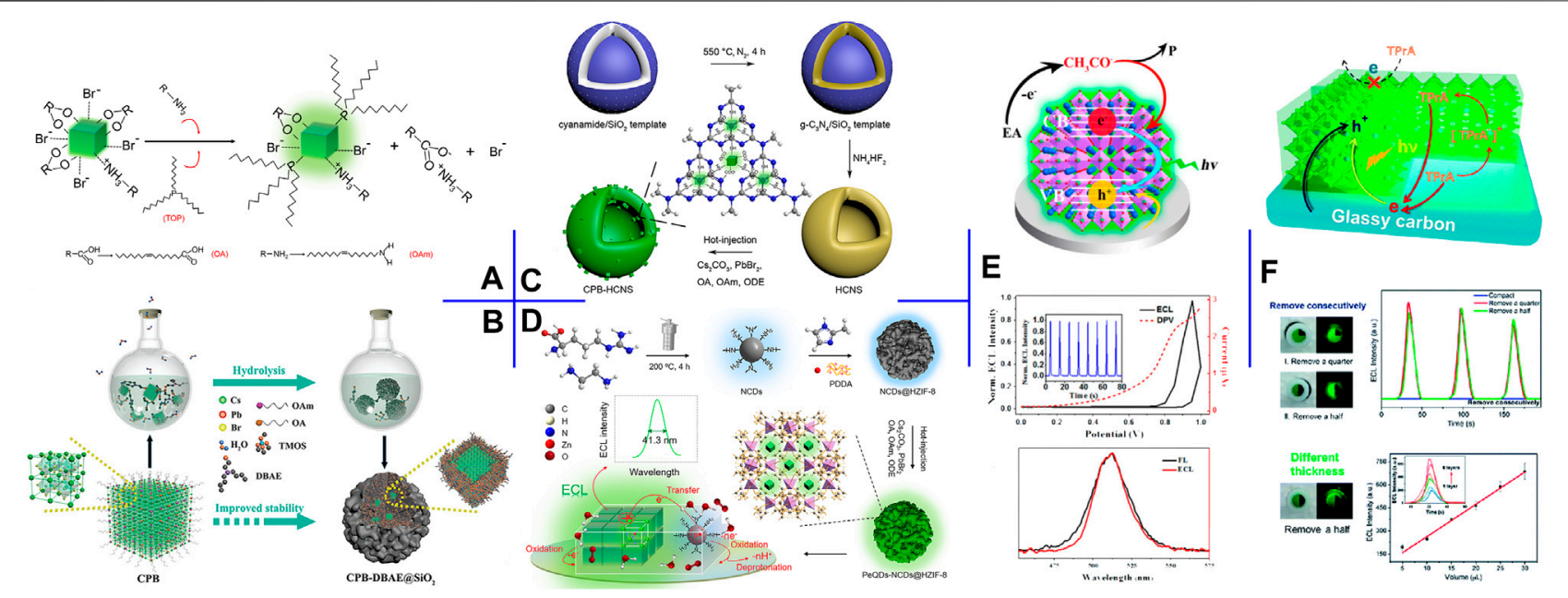

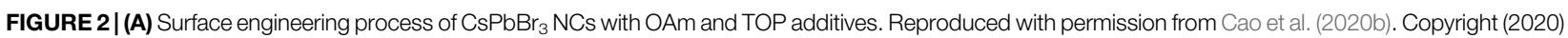
The Royal Society of Chemistry. (B) Schematic illustration of the preparation process of $\mathrm{CsPbBr}_{3}-\mathrm{DBAE} @ \mathrm{SiO}_{2}$ ternary hybrids. Reproduced with permission from Li et al. (2019). Copyright (2019) Wiley-VCH. (C) Schematic illustration of the construction process of $\mathrm{CsPbBr}_{3}-\mathrm{HCNS}_{\mathrm{S}}$ nanocomposite. Reproduced with permission from Cao et al. (2020c). Copyright (2020) American Chemical Society. (D) Schematic illustration of the construction process of CsPbBr 3 -NCDs@HZIF-8 nanocomposite. Reproduced with permission from Cao et al. (2020a). Copyright (2020) American Chemical Society. (E) ECL mechanism, the ECL-potential and DPV curves, and the PL and ECL spectra of the high-quality $\mathrm{CsPbBr}_{3}$ QDs film in EA. Reproduced with permission from Xue et al. (2017). Copyright (2017) American Chemical Society. (F) Schematic illustration of the three-phase heterostructure strategy and the corresponding ECL responses. Reproduced with permission from Qiu et al. (2019). Copyright (2019) The Royal Society of Chemistry.

and structural diversity. Cao et al. (2020a) designed a ternary nanocomposite by successively loading aminated carbon dots (NCDs) and $\mathrm{CsPbr}_{3}$ QDs in situ into the hierarchical zeolite imidazole framework-8 (HZIF-8) (Figure 2D). In this confined structure, HZIF-8 acted as a robust matrix for the loading of guest QDs and significantly enhanced the stability of $\mathrm{CsPbBr}_{3}$ QDs. Meanwhile, NCDs not only contained sufficient amines as efficient intra-nanomaterial coreactants of PeQDs for selfenhanced ECL but also improved the charge injection/transfer capacities of the hybrids. Consequently, the ternary architecture guaranteed high ECL performance in terms of stability and efficiency.

\section{INTERFACE INVESTIGATION AND MANIPULATION}

The present ECL study of PeNCs mainly focuses on their solidstate film. Thus, the interfacial reaction and manipulation are of paramount importance for better ECL performance. Cai et al. (2018) deposited an optimized amount of $\mathrm{CsPbBr}_{3}$ NCs hexane solution on glassy carbon electrode (GCE) by drop-casting. The cross-linked surface ligands (OA, OAm) could self-assemble a smooth and robust shell and facilitate superlattice formation, resulting in a strong and stable ECL signal in an aqueous solution.

To fabricate a high-quality $\mathrm{CsPbBr}_{3}$ QDs film, Xue et al. (2017) proposed a scraping coating method. In detail, $\mathrm{Cs} \mathrm{PbBr}_{3}$ QDs thick hexane slurry was dropped on GCE and then scraped with a glass rod. Afterward, the modified GCE was dipped into EA for $1 \mathrm{~s}$ several times. The established film showed reduced grain size and dense coverage, affording five-fold ECL efficiency in EA (vs.
$\left.\mathrm{Ru}(\mathrm{bpy})_{3}{ }^{2+} / \mathrm{TPrA}\right)$ and an ultranarrow fwhm of $24 \mathrm{~nm}$ (Figure 2E).

Solution-processed colloidal PeNCs exist flooded long-chain aliphatic ligands on their surface, which reduce the interface conductivity and affect the ECL property. Qiu et al. (2019) investigated the interfacial ECL behavior of $\mathrm{CsPbBr}_{3} \mathrm{NCs}$ film and proposed a three-phase heterostructure strategy for enhanced ECL intensity. The three-phase interface of GCE/ $\mathrm{CsPbBr}_{3} \mathrm{NCs}$ /acetonitrile was constructed by removing partial NCs film to expose the GCE substrate. They verified that the enhanced ECL intensity is closely related to the effective threephase interface rather than the exposed area of the GCE surface (Figure 2F).

\section{ELECTROCHEMILUMINESCENCE SENSING APPLICATION}

The ECL sensing application of PeNCs mainly focuses on the quantification of small biological molecules, which show a direct quenching or enhancing effect on the ECL signal. $\mathrm{H}_{2} \mathrm{O}_{2}$ was verified to be involved in the anodic charge transfer of $\mathrm{CsPbBr}$ NCs to produce efficient ECL in an aqueous solution. By using the GCE modified with $\mathrm{CsPbr}_{3} \mathrm{NCs}$, therefore, sensitive $\mathrm{H}_{2} \mathrm{O}_{2}$ sensing could be realized (Huang et al., 2017). Since AA is a powerful coreactant for $\mathrm{CsPbBr}_{3} \mathrm{NCs}$, an analogous configuration was also employed for AA detection (Cao et al., 2020b). Because alkaline phosphatase (ALP) can catalyze the production of AA from ascorbic acid 2-phosphate, the AA sensing platform could be further extended for ALP measurement (Wang et al., 2020b). Dopamine could be 
sensitively detected based on its effective quenching effect on the ECL of $\mathrm{CsPbBr}_{3}$ NCs/TPrA (Wang et al., 2020c) or $\mathrm{FAPbBr}_{3} /$ TPrA (Wang et al., 2020a) system.

Monochromatic ECL of PeNCs illustrates the great potential in building ECL resonance energy transfer (RET) systems. Cao et al. (2020c) synthesized dual-potential ECL emitter of $\mathrm{CsPbBr}_{3}$ NCs@HCNS. The anodic ECL of $\mathrm{CsPbBr}{ }_{3} \mathrm{NCs}$ was quenched by rhodamine $6 \mathrm{G}$ due to efficient ECL-RET, while the cathodic ECL of HCNS remained unchanged. Further combining with a DNA probe for CD44 receptors targeting and signal amplification via hybridization chain reaction (HCR), a ratiometric strategy was proposed for the sensitive and accurate evaluation of CD44 expression on MCF-7 cells. Cao et al. (2020a) also designed a 5'-hydroxyl terminal DNA probe, which successively underwent phosphorylation of T4 polynucleotide kinase (T4 PNK), cleavage of $\lambda$-exonuclease, signal amplification of HCR, and complementary capture of an electrode, a similar ECL-RET system between the self-enhanced $\mathrm{CsPbBr}_{3}$-NCDs@HZIF-8 ternary nanocomposite and rhodamine $6 \mathrm{G}$ was established for ultrasensitive T4 PNK activity evaluation.

\section{CONCLUSION AND OUTLOOKS}

This mini-review primarily focuses on the state of art of PeNCs in the ECL domain. The topics cover the emerging PeNCs emitters to date, the highlighting on the stabilization and interface manipulation strategies for desirable ECL performance, and a brief summary of the tentative sensing applications. In the past four years, various PeNCs, including fully-inorganic or organometallic lead-based PeNCs, bismuth-based PeNCs, and mixed-ions PeNCs are discovered with ECL activity and their underlying ECL mechanisms are investigated systematically. During the course of experiment, several unique ECL merits are disclosed, such as electrochemically switchable ECL property, ultranarrow ECL linewidth, and composition-tunable ECL performance. In order to meet the challenges in improving the stability and enhancing the charge injection/transfer capacities of PeNCs, elaborate strategies have been proposed, such as surface engineering, nanocomposite construction, and interface manipulation. As presented above, PeNCs are becoming promising alternatives to traditional semiconductor ECL transmitters for idiographic sensing applications.

Despite considerable progress, ECL research on PeNCs is still in its infancy, and huge challenges remain in exploring highperformance PeNCs luminophores and realizing their unique ECL applications. In terms of material synthesis, on the one hand, lead-based PeNCs can be further improved as promising ECL candidates through morphology control and surface engineering. For example, ligands with both co-reaction and stability

\section{REFERENCES}

Cai, Z., Li, F., Xu, W., Xia, S., Zeng, J., He, S., et al. (2018). Colloidal CsPbBr perovskite nanocrystal films as electrochemiluminescence emitters in aqueous solutions. Nano Res. 11, 1447-1455. doi:10.1007/s12274-017-1760-7 capabilities can be utilized for surface modification to achieve self-enhanced ECL property. Host-guest assemblies of functional ligands can tailor the highly-passivated NCs surface and unique ECL performance. The correlation between ECL property and the morphology of PeNCs has not been fully disclosed to date. On the other hand, exploring ECL-active lead-free PeNCs with environment friendliness is highly desired. However, their optical properties are still greatly inferior to lead-based counterparts. Thus, developing new synthetic approaches to access highly luminescent and stable lead-free PeNCs is crucial to their ECL progress. The soft ionic crystal structure and high defect tolerance of PeNCs determine the feasibility of ion exchange or doping. Hence, high-quality bimetallic or polymetallic PeNCs can be designed by doping analogous or impurity ions for tunable ECL property. Although the doping of $\mathrm{Rb}^{+}$and $\mathrm{Sb}^{3+}$ can boost the ECL intensity of PeNCs, the in-depth mechanism has not been fully elucidated, that is, for example, theoretical computations of energy level arrangement. In addition, the construction of PeNCs-devised nanocomposites is an alternative method, especially in exploring a suitable architecture for the trade-off between ECL efficiency and structural stability.

In terms of ECL applications, the facile PL tunability of PeNCs over the entire visible spectral region through halogen exchange has been universally recognized, but analogous multicolor ECL of PeNCs has not yet been fully achieved because of their relatively low ECL efficiency. The high ECL efficiency and color purity make lead-based PeNCs very attractive for ECL biosensing, but the toxicity issue of $\mathrm{Pb}^{2+}$ must be considered, and forming a compact and biocompatible coating might be a good choice. The stability of aqueous ECL remains to be addressed for reproducible analysis results. Nevertheless, it might be a new idea to utilize their instability or crystal conversion to realize some unique sensing applications. Also, their unique ECL properties can be further exploited and utilized, such as electrochemical switchability and monochromaticity, and a deeper understanding of PeNCs-related sensing mechanisms is also indispensable.

\section{AUTHOR CONTRIBUTIONS}

YC summarized them and wrote the article under the instruction of J-JZ.

\section{FUNDING}

This work was supported by the National Natural Science Foundation of China (Grant No. 21834004). 
self-sustaining suprastructure for electrochemiluminescence bioanalsis. Anal. Chem. 93, 1818. doi:10.1021/acs.analchem.0c04717

Cao, Y., Zhu, W., Li, L., Zhang, Z., Chen, Z., Lin, Y., et al. (2020b). Size-selected and surface-passivated $\mathrm{CsPbBr}_{3}$ perovskite nanocrystals for self-enhanced electrochemiluminescence in aqueous media. Nanoscale 12, 7321-7329. doi:10.1039/d0nr00179a

Cao, Y., Zhu, W., Wei, H., Ma, C., Lin, Y., and Zhu, J. J. (2020c). Stable and monochromatic all-inorganic halide perovskite assisted by hollow carbon nitride nanosphere for ratiometric electrochemiluminescence bioanalysis. Anal. Chem. 92, 4123-4130. doi:10.1021/acs.analchem.0c00070

Chen, L., Kang, Q., Li, Z., Zhang, B., Zou, G., and Shen, D. (2020). Tunable electrochemiluminescence properties of $\mathrm{CsPBr}_{3}$ perovskite nanocrystals using mixed-monovalent cations. New J. Chem. 44, 3323-3329. doi:10.1039/c9nj05665c

Ding, Z., Quinn, B. M., Haram, S. K., Pell, L. E., Korgel, B. A., and Bard, A. J. (2002). Electrochemistry and electrogenerated chemiluminescence from silicon nanocrystal quantum dots. Science 296, 1293-1297. doi:10.1126/science.1069336

Fu, K., He, Y., Zhang, B., Gao, X., and Zou, G. (2020a). Enhanced aqueous stability and radiative-charge-transfer of $\mathrm{CsPbBr} / \mathrm{Ag}_{2} \mathrm{~S}$ perovskite nanocrystal hybrids. J. Electroanal. Chem. 858, 113835. doi:10.1016/j.jelechem.2020.113835

Fu, L., Fu, K., Hsu, H. Y., Gao, X., and Zou, G. (2020b). Ce ${ }^{4+}$ doping to modulate electrochemical and radiative-charge-transfer behaviors of $\mathrm{Cs} \mathrm{PbBr}_{3}$ perovskite nanocrystal. J. Electroanal. Chem. 876, 114546. doi:10.1016/j.jelechem.2020. 114546

Hao, N., Lu, J., Dai, Z., Qian, J., Zhang, J., Guo, Y., et al. (2019). Analysis of aqueous systems using all-inorganic perovskite $\mathrm{CsPBr}_{3}$ quantum dots with stable electrochemiluminescence performance using a closed bipolar electrode. Electrochem. Commun. 108, 106559. doi:10.1016/j.elecom.2019.106559

Huang, Y., Fang, M., Zou, G., Zhang, B., and Wang, H. (2016). Monochromatic and electrochemically switchable electrochemiluminescence of perovskite $\mathrm{CsPbBr}_{3}$ nanocrystals. Nanoscale 8, 18734-18739. doi:10.1039/c6nr06456f

Huang, Y., Long, X., Shen, D., Zou, G., Zhang, B., and Wang, H. (2017). Hydrogen peroxide involved anodic charge transfer and electrochemiluminescence of allinorganic halide perovskite $\mathrm{CsPBr}_{3}$ nanocrystals in an aqueous medium. Inorg. Chem. 56, 10135-10138. doi:10.1021/acs.inorgchem.7b01515

Jia, J., Fu, K., Hou, S., Zhang, B., Fu, L., Hsu, H. Y., et al. (2019). Enhanced charge injection and recombination of $\mathrm{CsPbr}_{3}$ perovskite nanocrystals upon internal heterovalent subsitution. J. Phys. Chem. C 123, 29916-29921. doi:10.1021/acs. jpcc.9b10449

Kong, Y., Zhang, B. H., Zeng, Z. H., Zhang, Y. W., and Niu, L. (2020). Recent advances in electrochemiluminescence of halide perovskites. Chin. J. Anal. Chem. 48, e20021-e20031. doi:10.1016/S1872-2040(19)61218-1

Li, L., Zhang, Z., Chen, Y., Xu, Q., Zhang, J. R., Chen, Z., et al. (2019). Sustainable and self-enhanced electrochemiluminescent ternary suprastructures derived from $\mathrm{Cs} \mathrm{PbBr}_{3}$ perovskite quantum dots. Adv. Funct. Mater. 29, 1902533. doi:10. 1002/adfm.201902533

Li, Z., Kang, Q., Chen, L., Zhang, B., Zou, G., and Shen, D. (2020). Enhancing aqueous stability and radiative-charge-transfer efficiency of $\mathrm{CsPbBr}$ perovskite nanocrystals via conductive silica gel coating. Electrochim. Acta 330, 135322. doi:10.1016/j.electacta.2019.135332
Ma, C., Cao, Y., Gou, X., and Zhu, J. J. (2020). Recent progress in electrochemiluminescence sensing and imaging. Anal. Chem. 92, 431-454. doi:10.1021/acs.analchem.9b04947

Miao, W. (2008). Electrogenerated chemiluminescence and its biorelated applications. Chem. Rev. 108, 2506-2553. doi:10.1021/cr068083a

Peng, H., Wu, W., Huang, Z., Xu, L., Sheng, Y., Deng, H., et al. (2020). Cathodic electrochemiluminescence performance of all-inorganic perovskite $\mathrm{CsPBrr}_{3}$ nanocrystals in an aqueous medium. Electrochem. Commun. 111, 106667. doi:10.1016/j.elecom.2020.106667

Qiu, L., Lin, L., Huang, Y., Lai, Z., Li, F., Wang, S., et al. (2019). Unveiling the interfacial electrochemiluminescence behavior of lead halide perovskite nanocrystals. Nanoscale Adv. 1, 3957-3962. doi:10.1039/c9na00456d

Stoumpos, C. C., and Kanatzidis, M. G. (2016). Halide perovskites: poor man's high-performance semiconductors. Adv. Mater. 28, 5778-5793. doi:10.1002/ adma. 201600265

Tan, X., Zhang, B., and Zou, G. (2017). Electrochemistry and electrochemiluminescence of organometal halide perovskite nanocrystals in aqueous medium. J. Am. Chem. Soc. 139, 8772-8776. doi:10.1021/jacs.7b05073

Wang, X. Y., Wu, M. X., and Ding, S. N. (2020b). Anodic electrochemiluminescence from $\mathrm{CsPbBr}_{3}$ perovskite quantum dots for an alkaline phosphatase assay. Chem. Commun. 56, 8099-8102. doi:10.1039/ d0cc03648j

Wang, X., Yu, L., Kang, Q., Chen, L., Jin, Y., Zou, G., et al. (2020a). Enhancing electrochemiluminescence of $\mathrm{FAPbBr}_{3}$ nanocrystals by using carbon nanotubes and $\mathrm{TiO}_{2}$ nanoparticles as conductivity and co-reaction accelerator for dopamine determination. Electrochim. Acta 360, 136992. doi:10.1016/j. electacta.2020.136992

Wang, Y., Chen, T., Huang, C., Wang, Y., Wu, J., and Sun, B. (2020c). Electrochemically switchable electrochemiluminescent sensor constructed based on inorganic perovskite quantum dots synthesized with microwave irradiation. J. Electroanal. Chem. 867, 114181. doi:10.1016/j.jelechem.2020. 114181

Wusimanjiang, Y., Yadav, J., Arau, V., Steen, A. E., Hammer, N. I., and Pan, S. (2019). Blue electrogenerated chemiluminescence from halide perovskite nanocrystals. J. Anal. Test. 3, 125-133. doi:10.1007/s41664-018-0082-4

Xue, J., Zhang, Z., Zheng, F., Xu, Q., Xu, J., Zou, G., et al. (2017). Efficient solid-state electrochemiluminescence from high-quality perovskite quantum dot films. Anal. Chem. 89, 8212-8216. doi:10.1021/acs.analchem.7b02291

Conflict of Interest: The authors declare that the research was conducted in the absence of any commercial or financial relationships that could be construed as a potential conflict of interest.

Copyright () 2021 Cao and Zhu. This is an open-access article distributed under the terms of the Creative Commons Attribution License (CC BY). The use, distribution or reproduction in other forums is permitted, provided the original author $(s)$ and the copyright owner(s) are credited and that the original publication in this journal is cited, in accordance with accepted academic practice. No use, distribution or reproduction is permitted which does not comply with these terms. 